\title{
Magneto Hydrodynamic Calculation of Electromagnetic Stirring with Concave Shaped Free Surface Using Based Plane Shadow Method
}

\author{
Akira URITA* and Keisuke FUJISAKI \\ Toyota Technological Institute, 2-12-1 Hisakata, Tempaku, Nagoya, 468-8511 Japan. \\ (Received on September 26, 2017; accepted on November 24, 2017)
}

\begin{abstract}
The electromagnetic stirring (EMS) is a method to stir molten metal using in the steel making like the continuous casting. Physics concerning the EMS includes electromagnetic and fluid dynamics with free surface, which is magneto hydrodynamics. Theoretical analysis of magneto hydrodynamics is limited and/ or very difficult, so that the numerical computation is required to analyze magneto hydrodynamic phenomena. It means that Maxwell's equations and Navier-Stokes equations must be solved simultaneously, and a lot of calculation time is expected. In this study, "based plane shadow method", which is similar to the previous "R shadow method" but different from it in ability to be applied to any shape of the free surface, is suggested. Validity of the "based plane shadow method" is demonstrated by solving a magneto hydrodynamic problem simulating the continuous casting. It is shown that a comparable accuracy with solution obtained to solve the governing equations directly and a large amount of reduction in computational time achieve.
\end{abstract}

KEY WORDS: electromagnetic stirring; continuous casting; molten metal; free surface; magneto hydrodynamics; magneto hydrodynamic calculation; shadow method.

\section{Introduction}

There are many studies ${ }^{1-4)}$ in the steel making process applied with electromagnetic fields for the purpose of developing the seeds of new technique for high productivity and high quality. Continuous casting is a very important process which affects final quality of steel products in manufacturing process. Electromagnetic stirring (EMS) $)^{5,6)}$ is a method to stir molten metals in non-contact operation with electromagnetic forces generated by interaction of magnetic field and electric eddy current induced by the alternative magnetic field. The EMS is free from contaminations by giving some velocity to the molten steel and it can take a variety of configurations of stirring with various arrangements of electromagnetic actuators. Generation of segregation and attachment of $\mathrm{Al}_{2} \mathrm{O}_{3}$ particles makes quality of the steel be low. ${ }^{7)}$ Large amounts of energy and time are needed to remove them. This is one of the reasons that the EMS is used with the continuous casting. A free surface problem, which affects the quality of the products, occurs in the process because solidification begins beneath the surface of the molten metal. The shape of the free surface is concave in the EMS process because of the centrifugal force generated by rotation of the molten metal.

Temperature of the molten steel is more than $1500^{\circ} \mathrm{C}$, so that it is very difficult to measure the physical states of it. It

\footnotetext{
* Corresponding author: E-mail: urita@toyota-ti.ac.jp DOI: http://dx.doi.org/10.2355/isijinternational.ISIJINT-2017-564
}

is the reason that numerical analysis is required. In the free surface problem of the continuous casting, a boundary condition of the electromagnetic field at the free surface varies with modification of the surface shape by electromagnetic force. Magneto hydrodynamic treatment is required to analyze this problem. Maxwell's equations, which are governing equations of electromagnetic fields, and Navier-Stokes equations, which are ones of fluid fields, must be solved simultaneously to analyze this problem with high accuracy. Such calculations need a large amount of computational time, so that some numerical modeling must be considered.

One of the authors proposed an "R shadow method")" as a high efficient computation method of magneto hydrodynamic phenomena such as ones observed in the pulse electromagnetic casting (pulse EMC) processes ${ }^{3,9)}$ which give a convex shape of free surface of the molten metals, and has confirmed validity of the method. This method, however, cannot deal with a complex shape of free surface because the free surface shape is assumed to be monotone. On the other hand, the electromagnetic stirring, which is a method to give a rotation velocity to the molten metal, gives a concave shape of the free surface to the molten metals. New method is required to analyze such process because the "R shadow method" cannot treat a surface with general shape. In this paper, a "based plane shadow method", which is applicable to molten metals with arbitrary shape of free surfaces, is proposed, and validity and utility of this method is demonstrated through performing numerical analysis of molten metal with the EMS. 


\section{Unsteady Magneto Hydrodynamic Analysis}

In general, electromagnetic field and flow field need to be solved simultaneously in magneto hydrodynamic analysis. Computations are carried out with the J-MAG and the ANSYS Fluent for analyses of the electromagnetic and flow fields respectively.

\subsection{Electromagnetic Field Model}

Electromagnetic field is analyzed by solving Maxwell's equation represented in terms of the vector potential with finite element method. The $j \omega$ method, which replaces time differential operators with $j \omega$, is used here because it is a quasi-stationary state model. Electromagnetic field is assumed to change fast enough in comparison with fluid dynamics. Then the Maxwell's equation becomes as follows.

$$
\nabla \times\left([\dot{\mu}]^{-1} \nabla \times \dot{A}\right)+\sigma(j \omega \dot{A}+\nabla \dot{\phi})=0
$$

where $\boldsymbol{A}$ is vector potential, $\phi$ is scholar potential, $\mu$ is magnetic permeability, $\sigma$ is electrical conductivity, $\omega$ is angular frequency, and $j$ is the imaginary unit. Dot on the letter expresses complex number.

\subsection{Flow Field Model}

Flow field is analyzed by solving continuous equation and Navier-Stokes equation for incompressible flow considering the molten metal as incompressible fluid with finite volume method. Turbulent flow is computed with Large Eddy Simulation $^{10)}$ (LES).

$$
\operatorname{div} v=0
$$

$$
\rho \frac{\mathrm{D} \boldsymbol{v}}{\mathrm{D} t}=-\nabla p+\eta \Delta \boldsymbol{v}+\rho \boldsymbol{g}+\boldsymbol{F}_{e m}
$$

where $\boldsymbol{v}$ is flow velocity vector, $\rho$ is fluid density, $p$ is pressure, $\eta$ is dynamic viscosity, $\boldsymbol{g}$ is acceleration vector of the gravity, and $\boldsymbol{F}_{e m}$ is external force resulting from electromagnetic field. This force is expressed as follows.

$$
\boldsymbol{F}_{e m}=\sigma\left(\frac{\partial \boldsymbol{A}}{\partial t}+\nabla \phi\right) \times(\nabla \times \boldsymbol{A})
$$

The shape of free surface of molten metal is evaluated with Volume of Fluid ${ }^{11}$ (VOF) method. Following advective equation is solved in the VOF method

$$
\frac{\mathrm{D} F}{\mathrm{D} t}=0
$$

where $F$ is ratio of liquid which takes the value of 0 to 1 .

\subsection{Direct Method}

Figure 1 shows a schematic of magneto hydrodynamic computation using a direct method. At first, the electromagnetic field induced by impressed magnetic field in the molten metal and resulting electromagnetic force is calculated numerically by solving Maxwell's equation without considering any fluid motion. Next, the fluid field is calculated with the electromagnetic force as external force inducing fluid motion and deformed fluid surface is evaluated with the VOF method. And then, return the first step with new situation such as new shape of fluid surface. Repeating this procedure gives the motion and properties of the electromagnetic fluid. It is, however, very expensive to solve three dimensional electromagnetic and fluid equations simultaneously. So "shadow methods", which are described in the next sections, are introduced to reduce the computational time without degeneracy of the numerical accuracy.

\subsection{R Shadow Method ${ }^{8}$}

Figure 2 illustrates the concept of the " $R$ shadow method". Electromagnetic force on a point which is moved to $\mathrm{P}_{1}$ after deformation by initial electromagnetic force is estimated as the same force acting before the deformation on a point $\mathrm{P}_{0}$. The point $\mathrm{P}_{0}$ is related with $\mathrm{P}_{1}$ by Eq. (6). Points $\mathrm{Q}_{0}$ and $\mathrm{Q}_{1}$ are intersections on the free surface with horizontal lines passing on the points $\mathrm{P}_{0}$ and $\mathrm{P}_{1}$ respectively.

$$
\mathrm{P}_{0} \mathrm{Q}_{0}=\mathrm{P}_{1} \mathrm{Q}_{1}
$$

But this method cannot give a valid force when free surface has i) wavy form or ii) a temporary change in a sign of the incline, or iii) when a point under consideration has no

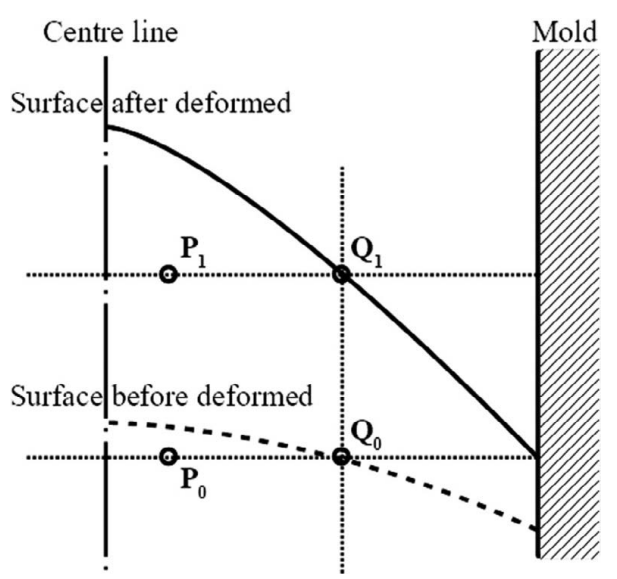

Fig. 2. The R shadow method.

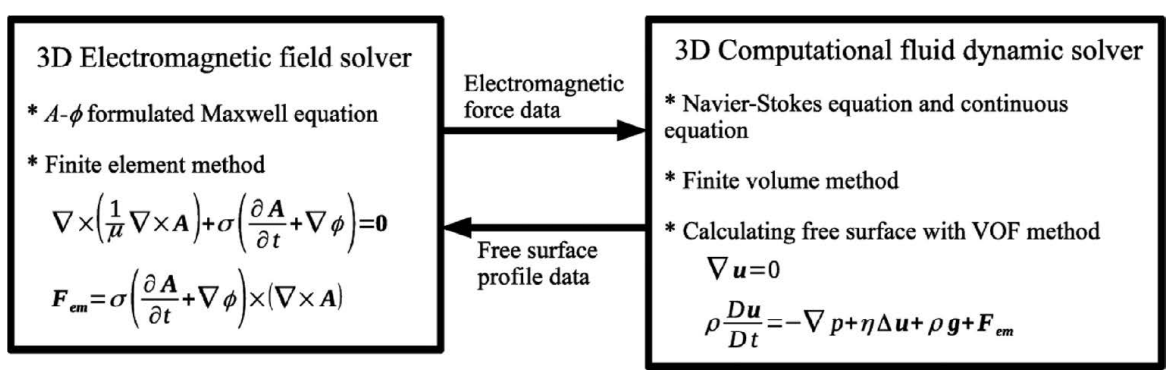

Fig. 1. Schematic diagram of magneto hydrodynamic computation using the direct method. 


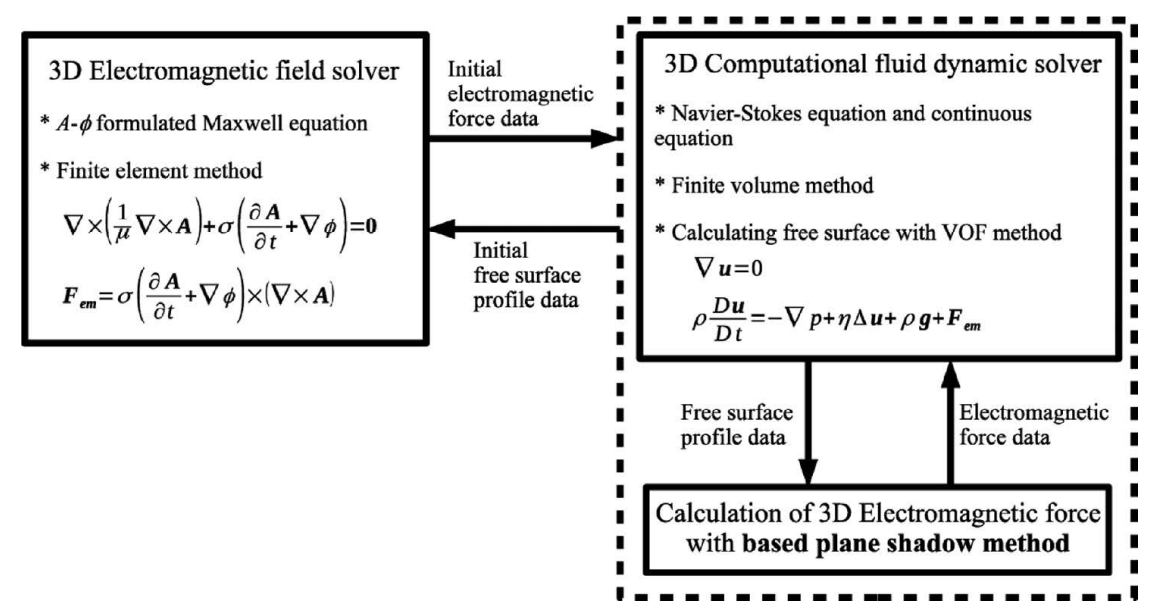

Fig. 3. Schematic diagram of magneto hydrodynamic computation using based plane shadow method.

surface in the same level. The forces act on deeper points than any surface also cannot estimate with this method.

\subsection{Based Plane Shadow Method ${ }^{12)}$}

Figure 3 shows schematic of magneto hydrodynamic computation using a "based plane shadow method" proposed in this paper. The electromagnetic field induced by impressed magnetic field in the molten metal and resulting electromagnetic force is calculated numerically, and then the fluid field and deformed fluid surface are calculated with the electromagnetic force in the same manner as the direct method. After that, the electromagnetic force with new shape of the free surface is estimated using the "based plane shadow method" without solving Maxwell's equation. The fluid field and the shape of the fluid surface are calculated with the new electromagnetic force. Time development of the electromagnetic and fluid phenomena is computed by repeating above procedure. The "based plane shadow method" is a procedure which estimates distribution of the electromagnetic force after deformation of the fluid using the distribution of the electromagnetic force before the deformation as well as the "R shadow method". Just one time calculation of the electromagnetic force is required at the start of computation, and large reduction of computational time can be expected.

Figure 4 illustrates the concept of the "based plane shadow method". A horizontal plane is taken as a based plane far from the surface of the molten metal. Electromagnetic force on a point which is moved to $\mathrm{P}_{1}$ after deformation by initial electromagnetic force is estimated as the same force acting before the deformation on a point $\mathrm{P}_{0}$. The point $P_{0}$ is related with $P_{1}$ by Eq. (7). Points $Q_{0}$ and $Q_{1}$ on a vertical line through $P_{1}$ are intersections of this line with the fluid surfaces before and after the deformation. Point $\mathrm{B}$ on the vertical line through $\mathrm{P}_{1}$ is also an intersection of this line with the based plane.

$$
\mathrm{BQ}_{0}: \mathrm{BQ}_{1}=\mathrm{BP}_{0}: \mathrm{BP}_{1}
$$

Electromagnetic force with any surface shape except cornice can be estimated with this method.

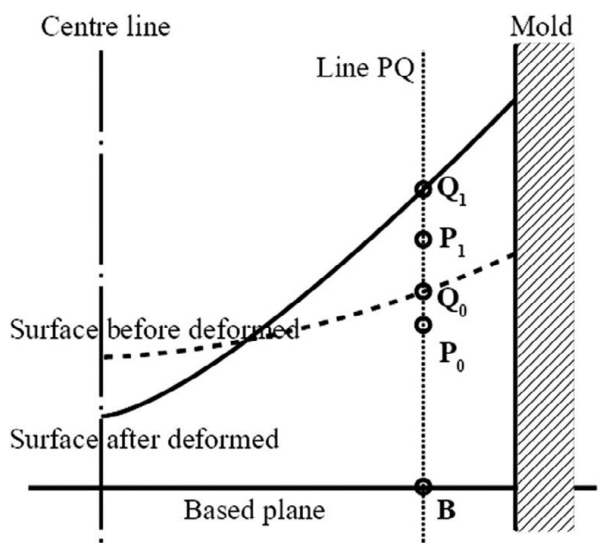

Fig. 4. Based plane shadow method.

\section{Calculation Results and Discussion}

\subsection{Analytic Model for Numerical Simulation}

Numerical simulation is performed for the in-mold EMS in the continuous casting process. A model arrangement used in this analysis and specifications of the stirring coils are shown in Fig. 5 and Table 1, respectively. Dimensions of the fluid region are $700 \mathrm{~mm}$ in height and $40 \mathrm{~mm}$ in radius. Nozzle to pour the molten metal is $125 \mathrm{~mm}$ in length and $6 \mathrm{~mm}$ in radius and settled from the top of the region. Free surface of the liquid metal is initially placed at $100 \mathrm{~mm}$ and the based plane is at $150 \mathrm{~mm}$ below the top. The based plane is assumed to be at the same height of centre of the coils. The electromagnetic and the fluid computations are performed within the azimuthal range of 180 and 10 degrees respectively assuming axisymmetry. The liquid metal is assumed as Ga whose melted temperature is $29.8^{\circ} \mathrm{C}$. Physical properties used in the numerical analysis are summarized in Table 2.

\subsection{Results of the Numerical Simulation}

\subsubsection{Electromagnetic Field}

Distribution of the electromagnetic force induced by the external magnetic field in the vertical plane at the initial condition in which the fluid is rest is illustrated in Fig. 6. Figure 7 shows distribution of electromagnetic force at $t=30$ secs., by which it can be considered that steady state has been realized, computed by the direct method and 


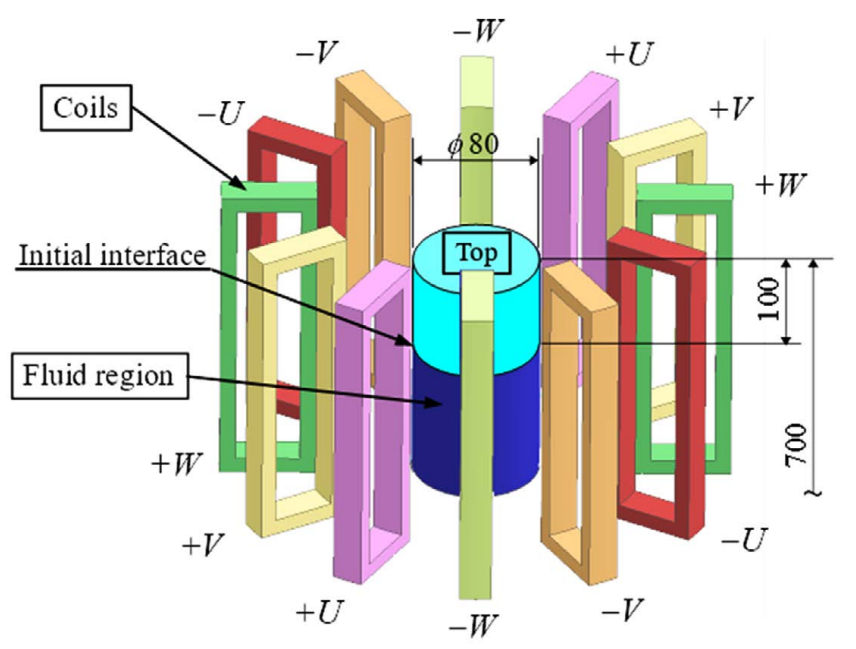

(a) Outline

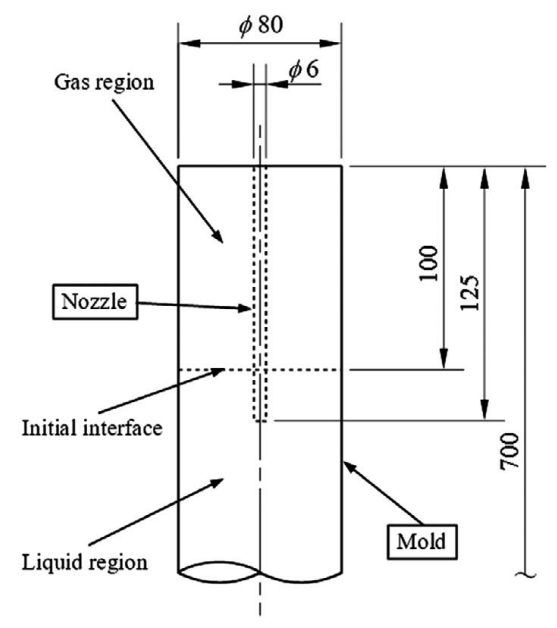

(b) Details near the interface

Fig. 5. Schematic of the computed object. (Online version in color.)

Table 1. Specifications of coils to generate electromagnetic force.

\begin{tabular}{cc}
\hline Frequency $(\mathrm{Hz})$ & 45 \\
Winding number & 60 \\
Phase current $\left(\mathrm{A}_{\mathrm{rms}}\right)$ & 25 \\
\hline
\end{tabular}

Table 2. Physical properties applied to the computation.

\begin{tabular}{clccc}
\hline & $\mu_{\mathrm{r}}$ & $\sigma[\mathrm{S} / \mathrm{m}]$ & $\rho\left[\mathrm{kg} / \mathrm{m}^{3}\right]$ & $\eta[\mathrm{Pa} \cdot \mathrm{s}]$ \\
\hline $\mathrm{Ga}$ & 1.0 & $3.85 \times 10^{6}$ & 6114 & 0.002 \\
Air & 0 & 0 & 1.205 & $1.822 \times 10^{-5}$ \\
\hline
\end{tabular}

the "based plane shadow method". The distribution of the electromagnetic force in the molten metal with the concave shape of the free surface can be estimated accurately by the "based plane shadow method".

\subsubsection{Fluid Field}

The fluid dynamic computation of the molten metal is carried out using the estimated electromagnetic force given by the "based plane shadow method" applying to the electromagnetic force obtained in each time steps. Non-slip

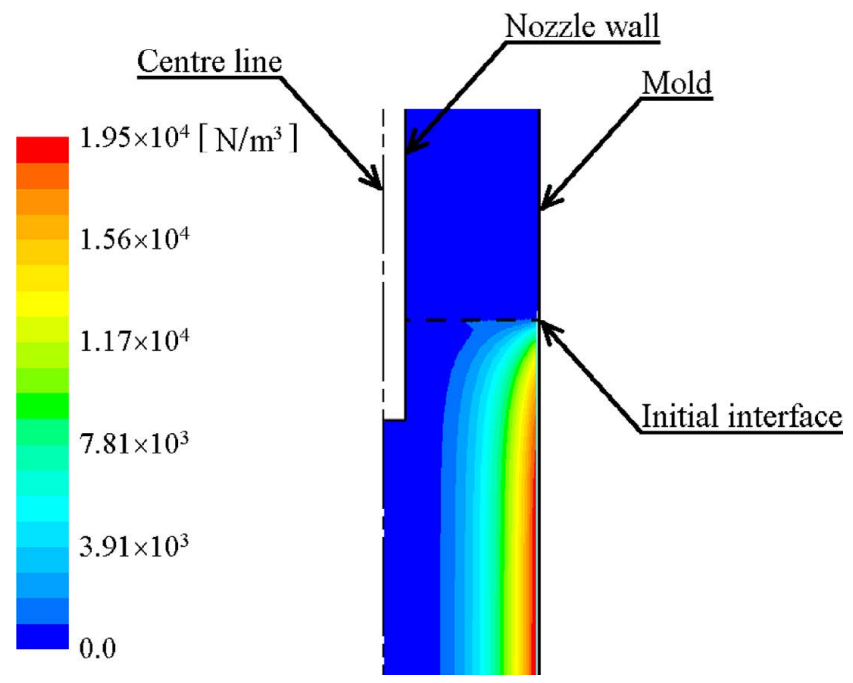

Fig. 6. Initial distribution of electromagnetic force in vertical plane. (Online version in color.)

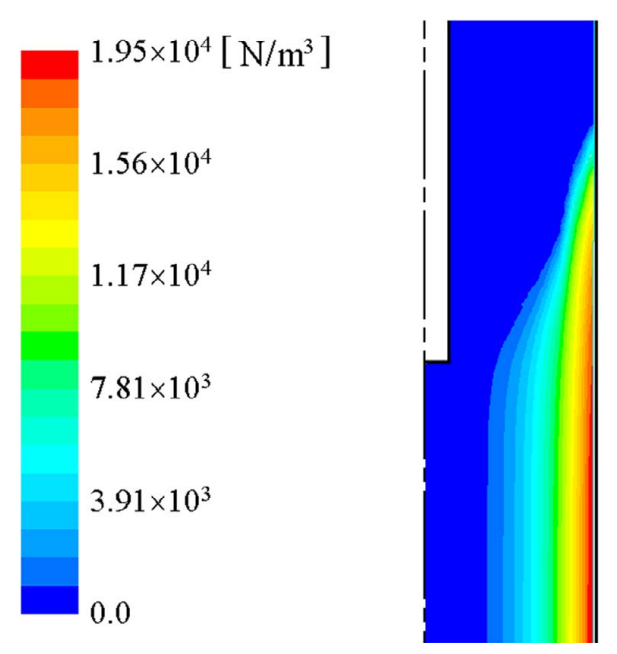

(a) direct method

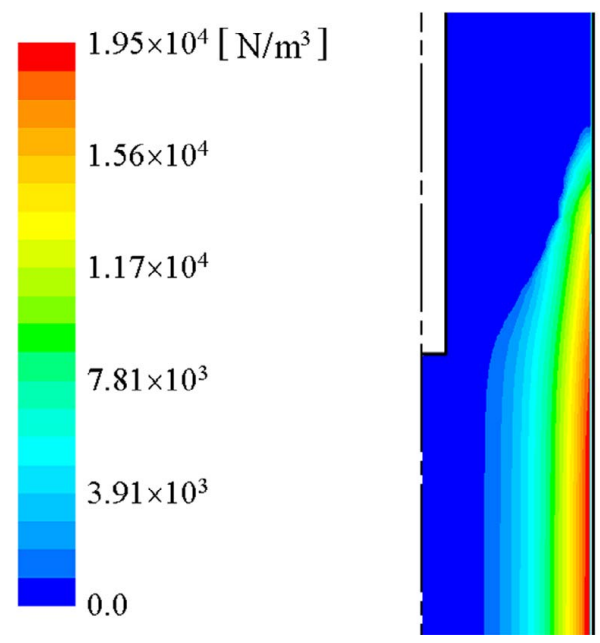

(b) based plane shadow method

Fig. 7. Estimated distributions of electromagnetic force in the vertical plane at steady state $(t=30 \mathrm{secs})$. (Online version in color.)

condition is applied on the walls. Flow ratio of the molten metal is assumed zero for simplicity and the nozzle exit and the outlet of the cast are treated in the same manner the other 


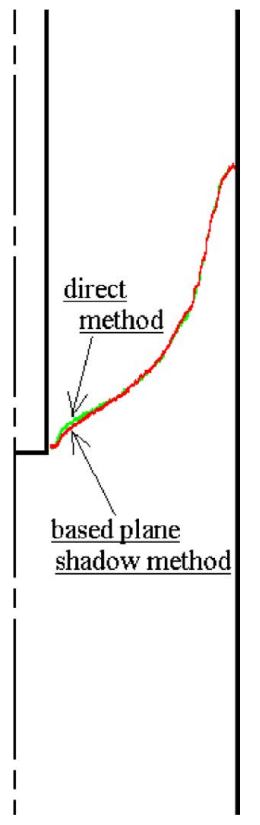

Fig. 8. The shapes of the free surface at steady state $(t=30 \mathrm{secs})$. (Online version in color.)

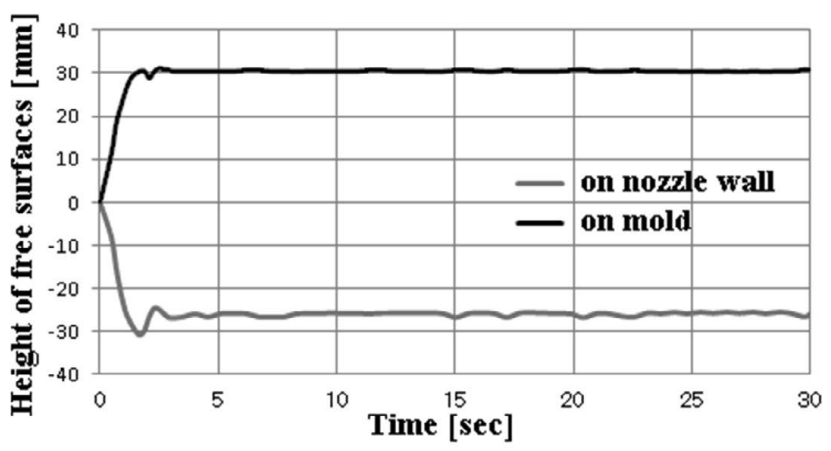

(a) direct method

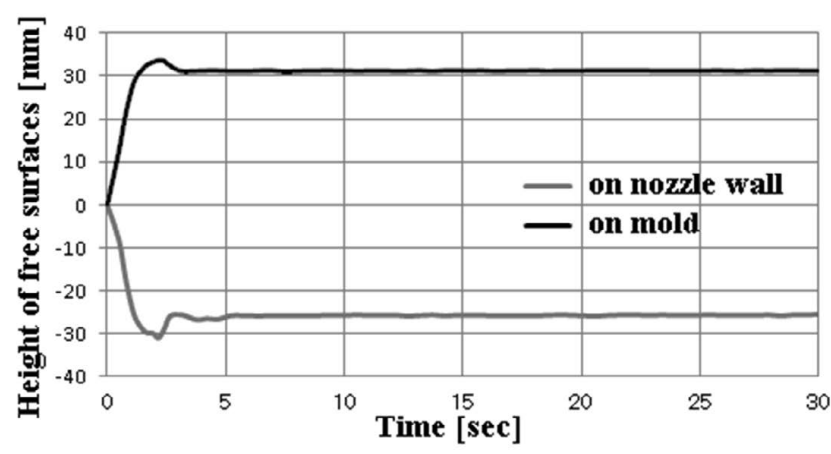

(b) based plane shadow method

Fig. 9. Time variation of the heights of the free surface on the nozzle and the outer walls. ordinary walls are done. Free surface is given as a surface with a constant pressure of atmospheric pressure. Temperature of the fluid is considered as a constant and it is used only for calculations of fluid properties. Figure 8 shows the shapes of the free surfaces calculated with the "based plane shadow method" (red line) and the direct method (green line) at $t=30 \mathrm{secs}$. The obtained shape of the free surface of the molten metal is almost the same as the one given by the direct method.

Figure 9 shows time variation of the heights of the free surface on the nozzle wall and on the outer wall. Both the direct method and the "based plane shadow method" achieve the steady states within 10 seconds and the heights

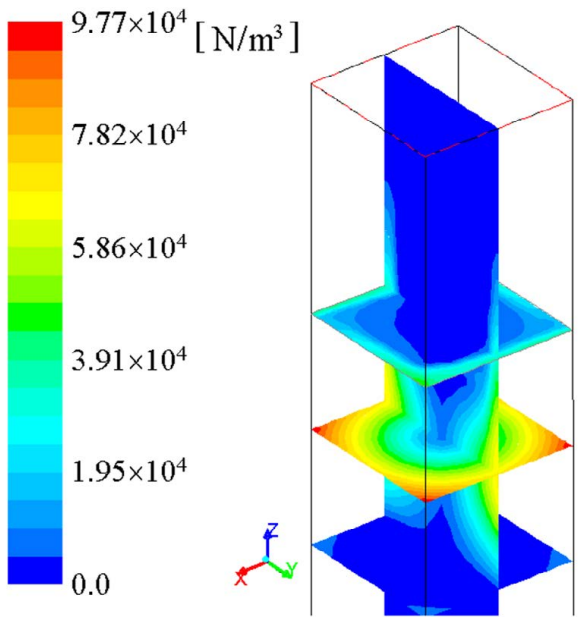

(a) electromagnetic force

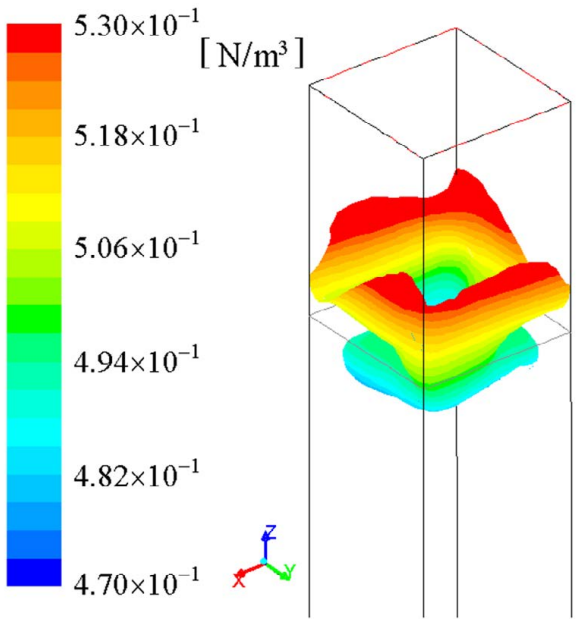

(b) shape of free surface

Fig. 10. Results of a case with a rectangular section using the "based plane shadow method". (Online version in color.)

Table 3. Computational time of the direct method and the "based plane shadow method".

\begin{tabular}{|c|c|c|c|c|c|}
\hline & \multicolumn{3}{|c|}{ Electromagnetic force } & \multirow{2}{*}{$\begin{array}{l}\text { Flow field } \\
\text { time }[\mathrm{min}]\end{array}$} & \multirow{2}{*}{$\begin{array}{c}\text { Total } \\
\text { computational } \\
\text { time }[\mathrm{min}]\end{array}$} \\
\hline Method & $\begin{array}{c}\text { Time } \\
{[\mathrm{min} / \mathrm{job}]}\end{array}$ & Frequency & $\begin{array}{l}\text { Subtotal of } \\
\text { computational } \\
\text { time }[\mathrm{min}]\end{array}$ & & \\
\hline Direct method & 5 & $\begin{array}{c}30 \\
\text { (every one second) }\end{array}$ & 150 & 30 & 180 \\
\hline $\begin{array}{c}\text { Based plane } \\
\text { shadow method }\end{array}$ & 5 & 1 & 5 & 30 & 35 \\
\hline
\end{tabular}


of the free surface vary similarly in the both cases although a very small difference is observed around $t=2$ secs. It means that the "based plane shadow method" provide not only accurate steady analysis but also accurate unsteady one. Table 3 indicates computational time both of the direct method and the "based plane shadow method". It shows that the "based plane shadow method" takes about 20 percent of computational time of the direct method. This fact means that the "based plane shadow method" which is proposed in this paper can treat magneto hydrodynamic phenomena such as EMS very efficiently without losing the accuracy.

\subsubsection{Case with a Rectangular Section}

Figure 10 displays the distributions of the electromagnetic force and the shape of the free surface computed with the "based plane shadow method" in the similar situation described previous sections except the cross section of a mold being rectangle. In this case, fluid computation must be performed at least within the azimuthal range of 90 degrees. So the ratio of total computational time of the "based plane shadow method" to that of the direct method should be more than the axisymmetric case. The computational time, however, can be estimated around 60 percent of that of the direct method, and it is still large amount of reduction.

\section{Conclusion}

Numerical analysis for the continuous casting of steel with the electromagnetic stirring (EMS) requires to solve governing equations of electromagnetic field and fluid field simultaneously, and it takes large amount of computational time. The "R shadow method" with which electromagnetic field is solved once at first and fluid field is calculated with the resulting electromagnetic force projected on the deformable fluid body has been proposed. This method can reduce the computational time but it cannot be applied to the case that the fluid has a surface with complex shape. In this paper, the "based plane shadow method" which can be applicable to the fluid with a more general surface shape is proposed, and its validity and utility is verified. Conclusions can be summarized as followings:

(1) Distribution of the electromagnetic force in the fluid with a concave surface can be estimated accurately using the "based plane shadow method".

(2) The shape of the fluid surface resulting from the electromagnetic force and the fluid motion can be reconstructed with the present method.

(3) Time variation of the shape of fluid surface can be simulated with the present method.

(4) Computational time with the "based plane shadow method" is reduced to about 20 percent of that with the direct method.

As a next subject, the influence of the height of the based plane will be investigated.

\section{Acknowledgment}

This work was supported by the Iron and Steel Institute of Japan Research Promotion Grant (H24-010). The authors are grateful to Dr. S. Satou for collaborating in execution of the computation.

\section{REFERENCES}

1) D. Li, Z. Su, J. Chen, Q. Wang, Y. Yang, K. Nakajima, K. Marukawa and J. He: ISIJ Int., 53 (2013), 1187.

2) K. Iwai and A. Maruyama: Jpn. J. Multiph. Flow, 30 (2016), 282 (in Japanese).

3) K. Fujisaki: IEEE Trans. Ind. Appl., 39 (2003), 1442.

4) K. Fujisaki: IEEE Trans. Magn., 39 (2003), 3541.

5) K. Fujisaki, K. Wajima and T. Ohki: IEEE Trans. Magn., 36 (2000), 1325 .

6) K. Fujisaki: IEEE Trans. Ind. Appl., 37 (2001), 1098.

7) N. Kochi, Y. Ueda, T. Uemura, T. Ishii and M. Iguchi: ISIJ Int., 51 (2011), 1011.

8) K. Fujisaki and T. Ueyama: J. Appl. Phys., 83 (1998), 6356.

9) N. Tani, K. Tanaka, S. Fukunaga, T. Toh, K. Tunenari, K. Umetsu, K. Hayashi and M. Zeze: Int. J. Cast Met. Res., 22 (2009), 298.

10) J. Smagorinsky: Mon. Weather Rev., 91 (1963), 99.

11) C. W. Hirt and B. D. Nichols: J. Comput. Phys., 39 (1981), 201.

12) K. Fujisaki: CAMP-ISIJ, 26 (2013), 166, CD-ROM (in Japanese). 